\title{
Strange hadron yields and ratios in heavy ion collisions at RHIC energy
}

\author{
G. Hamar ${ }^{a}$, L.L. Zhu ${ }^{b}$, P. Csizmadia ${ }^{a}$, and P. Lévai ${ }^{a}$ \\ ${ }^{a}$ RMKI Research Institute for Particle and Nuclear Physics, \\ P.O. Box 114, Budapest, 1525, Hungary \\ ${ }^{b}$ Institute of Particle Physics, Hua-Zhong Normal University, \\ Wuhan 430079, P.R. China
}

\begin{abstract}
Recent experimental data support the presence of quark coalescence in heavy ion collisions at RHIC energies. Hadronization of quark matter and hadron formation in heavy ion collisions can be described by the coalescence process, and measured data are reproduced successfully. On the other hand, the theoretical coalescence calculations are based on a non-relativistic description. Here we investigate the robustness of the coalescence description, using different wave-function overlap during hadron formation.
\end{abstract}

Quark coalescence has been proposed many years ago to describe quark matter hadronization [1, 2, 3. The basic idea was to consider massive constituent quarks in the deconfined phase, which are ready to hadronize through "coalescence", which is a clustering process driven by an attractive force between the properly coloured quark degrees of freedom. The presence of these massive excitations in the quarkmatter phase is supported by the analysis of lattice QCD data and the recognition of massive quasi-particles in the strongly interacting deconfined phase close to the quarkhadron phase transition [4. The attractive force generated by the gluons (which are considered in this way) is modelled by non-relativistic colour potential between the quarks and antiquarks. Mesons are produced by quark-antiquark coalescence. Baryons are produced in two steps: at first $\overline{3}$ diquarks appear through quark-quark coalescence, which is followed by a diquark-quark coalescence into a colourless baryon.

Although particle yields, ratios and spectra have been reproduced successfully in ALCOR [5, 6] and MICOR 7, 8, coalescence models, but thermal models were similarly successful in the low- $p_{T}$ region and more widely used because of their simplicity. At RHIC energy intense data collection has been performed in the intermediate- $p_{T}$ region $\left(3<p_{T}<8 \mathrm{GeV} / \mathrm{c}\right)$ and the measured anomalous proton/pion ratio could have been explained by quark coalescence and recombination models 9, 10, 11. This fact increased the interest in this microscopical hadronization mechanism and more applications appeared. The recognition of valence quark number scaling in the data on asymmetric flow $\left(v_{2}\right)$ supported very strongly the quark matter formation and quark coalescence at RHIC and SPS energies [12].

The success of the quark coalescence model raised an interesting question, namely why this model is working successfully, when it is based on quantum mechanics and non-relativistic quark-quark interaction picture. The main reason is the following: although the quarks are moving with a relativistic velocity out of the reaction volume, but they can participate in the coalescence process only if their relative velocity is small. In this case quantum mechanics becomes valid in the comoving system and wave functions start to play a significant role in the description of hadron formation. 
In this paper we investigate the sensitivity of the hadron yields on the use of the different quark and hadron wave functions. We will consider plain wave and gaussian wave functions for quarks and antiquarks, where the gaussian choice indicates the presence of a finite volume deconfined quark matter. For mesons we use the same two choices and extend the list with the hydrogen-like wave function, which is connected to an analogy between electromagnetic and strong forces with proper coupling constant. For diquarks we use the same three choices. However, the diquark and the plasma state may interact and the diquark wave function can be modified, flipping between the plain wave, gaussian and hydrogen like wave functions. Thus baryons are produced similarly to mesons, but in two steps, as we will discuss it in details.

At first we determine the quark coalescence cross section in quantum mechanics. We use the quantum mechanical pick-up reaction [13: $q_{1}+\left.Q\right|_{q_{2}} \longrightarrow h+Q^{\prime}$, where quark $q_{1}$ picks up quark $q_{2}$ from the plasma $Q$, producing prehadron $h$ and plasma $Q^{\prime}$. The quantum mechanical amplitude of the pick up reaction is given:

$$
g_{g h}=V_{g} \frac{-M_{h, Q^{\prime}}}{2 \pi} \int d^{3} \vec{x}_{1} d^{3} \vec{x}_{2} \widetilde{\Psi}^{*}\left(\vec{x}_{1}, \vec{x}_{2}\right) V\left(\vec{x}_{1}-\vec{x}_{2}\right) \phi_{1}\left(\vec{x}_{1}\right) \phi_{2}\left(\vec{x}_{2}\right),(1)
$$

where $\phi_{i}\left(\vec{x}_{i}\right)$ is the wave function of the $q_{i}$ quark, and $\widetilde{\Psi}\left(\vec{x}_{1}, \vec{x}_{2}\right)$ belongs to the prehadron with mass $M_{h}$. $M_{h, Q^{\prime}}$ is the reduced mass of $h$ and $Q^{\prime}$. Since $M_{h} \ll M_{Q^{\prime}}$ therefore $M_{h, Q^{\prime}} \simeq M_{h}$. The standard two-body coordinates can be introduced as relative distance vector $(\vec{r})$, relative momentum vector $(\vec{k})$, space and momentum vector of the center of mass $(\vec{X}, \vec{P})$. Since the outgoing prehadron has the momentum $\vec{P}=\vec{p}_{1}+\vec{p}_{2}$, thus the wave function $\widetilde{\Psi}$ is simplified to $\Psi(\vec{r}) \cdot e^{i \vec{P} \vec{X}}$.

In eq. (11) $V\left(\vec{x}_{1}-\vec{x}_{2}\right)$ denotes the quark-quark interaction. Here we introduce the Yukawa-potential, which depends on the relative distance, $r$ :

$$
V(r)=-\alpha\left\langle\lambda_{i} \lambda_{j}\right\rangle \frac{e^{-m_{g} r}}{r}
$$

The screening mass has a relatively large value at $T \approx T_{c}: m_{g}=0.8 \mathrm{GeV}$ [4]. The colour factor $\left\langle\lambda_{i} \lambda_{j}\right\rangle$ is determined by the colour combination of the interacting particles. In the limit of $m_{g}=0$ Coulomb potential is restored, which has been used in the ALCOR model [1]. In the MICOR model a Yukawa potential has been considered [7].

In $2 \rightarrow 2$ reactions (e.g. $a+b \rightarrow c+d$ ) the cross section can be determined from the amplitude as

$$
\sigma(k)=\frac{v_{c d}}{v_{a b}}\left|g_{g h}(k)\right|^{2},
$$

where $v_{a b}$ and $v_{c d}$ are the relative velocities. In our case the factor $v_{c d}$ could be large, because it is the relative velocity of the outgoing (pre)hadron and the plasma. Thus we have two choices during evaluation of eq. (3):

$$
\begin{aligned}
\left.\sigma(k) v_{q_{1} q_{2}}\right|_{\text {classical }} & =\frac{P}{M_{h}}\left|g_{g h}(k)\right|^{2} \\
\left.\sigma(k) v_{q_{1} q_{2}}\right|_{\text {relativistic }} & =\frac{P}{\sqrt{P^{2}+M_{h}^{2}}}\left|g_{g h}(k)\right|^{2}
\end{aligned}
$$

Now we are ready to introduce quark and prehadron wave functions to calculate numerically applicable coalescence cross sections from eqs. (11)-(5). In the lack of precise quark wave functions, we will use simple functions, which will be displayed and discussed after introducing the calculation of hadron production rates and yields. 
Previously, the coalescence cross sections were obtained from quantum mechanics. The wanted hadron production rates and yields can be determined by a statistical method based on rate equations using the former cross sections.

Prehadron $h$ is composed from quarks $q_{1}$ and $q_{2}$ via coalescence, and its production is proportional to the densities of the constituents, $n_{1}$ and $n_{2}$ [1, 17]:

$$
\partial_{\mu}\left(n_{h} u^{\mu}\right)=\left\langle\sigma_{12}^{h} v_{12}\right\rangle n_{1} n_{2} .
$$

The rate, $\left\langle\sigma_{12}^{h} v_{12}\right\rangle$, is calculated as a phase space average:

$$
\left\langle\sigma_{12}^{h} v_{12}\right\rangle=\frac{\int d^{3} \vec{p}_{1} d^{3} \vec{p}_{2} d^{3} \vec{x}_{1} d^{3} \vec{x}_{2} \rho_{12}\left(\vec{x}_{1}, \vec{x}_{2}\right) f_{1}\left(\vec{x}_{1}, \vec{p}_{1}\right) f_{2}\left(\vec{x}_{2}, \vec{p}_{2}\right) \sigma v_{12}}{\int d^{3} \vec{p}_{1} d^{3} \vec{p}_{2} d^{3} \vec{x}_{1} d^{3} \vec{x}_{2} \rho_{12}\left(\vec{x}_{1}, \vec{x}_{2}\right) f_{1}\left(\vec{x}_{1}, \vec{p}_{1}\right) f_{2}\left(\vec{x}_{2}, \vec{p}_{2}\right)}(7)
$$

where $f_{i}\left(\vec{x}_{i}, \vec{p}_{i}\right)$ are the quark momentum distributions and $\rho$ describes the locality of the quark coalescence. Requiring that quarks with the same location are able to coalesce, $\rho$ becomes a simple Dirac delta. Assuming isotrop plasma state, eq.(77) is simplified into the following expression:

$$
\left\langle\sigma_{12}^{h} v_{12}\right\rangle=\frac{\int d^{3} \vec{p}_{1} d^{3} \vec{p}_{2} f_{q}\left(m_{1}, \vec{p}_{1}\right) f_{q}\left(m_{2}, \vec{p}_{2}\right) \sigma v_{12}}{\int d^{3} \vec{p}_{1} d^{3} \vec{p}_{2} f_{q}\left(m_{1}, \vec{p}_{1}\right) f_{q}\left(m_{2}, \vec{p}_{2}\right)}
$$

During the evaluation of the rate one can use any proper distribution function. Because of the massive quarks we can use non-relativistic Boltzmann distribution:

$$
f_{q}^{\text {Boltzmann }}(m, \vec{p})=e^{-\frac{p^{2}}{2 m T}}
$$

In parallel, relativistic quark distribution can be used also, namely Jüttner distribution, which is simplified in the local rest frame of the expanding fireball:

$$
f_{q}^{J u t t n e r}(m, \vec{p})=e^{-\frac{u_{\mu} p^{\mu}}{T}}=e^{-\frac{\sqrt{p^{2}+m^{2}}}{T}}
$$

We will use both distributions and investigate the sensitivity of the rate on this choice and the temperature $T$. Furthermore, in the above quark momentum distribution functions we will use quark masses $m_{q}=0.3 \mathrm{GeV}$ and $m_{s}=0.5 \mathrm{GeV}$, which values are verified in the analysis of the lattice data close to the quark-hadron phase transition [4].

Now we have all expression to investigate prehadron production from quark matter. If we define the necessary wave functions for quarks and prehadrons, then we can perform numerical calculations and determine the wanted particle productions. As we mentioned earlier, we introduce two types of wave functions for quarks:

$$
\begin{array}{lr}
\phi_{i}=\frac{1}{\sqrt{V_{q}} e^{i \vec{p}_{i} \vec{x}_{i}}} & \text { PW }: \text { plain wave } \\
\phi_{i}=\frac{1}{\left(2 \pi \rho^{2}\right)^{3 / 4}} e^{-\frac{x_{i}^{2}}{4 \rho^{2}}} e^{i \vec{p}_{i} \vec{x}_{i}} & \mathrm{G}: \text { gaussian }
\end{array}
$$

For prehadrons we introduce the following wave functions:

$$
\begin{array}{ll}
\widetilde{\Psi}=\frac{1}{\sqrt{V_{f}}} \frac{1}{\sqrt{V_{h}}} e^{i \vec{P} \vec{X}} & \mathrm{PW}: \text { plain wave } \\
\widetilde{\Psi}=\frac{1}{\sqrt{V_{f}}} \frac{1}{\sqrt{\pi a^{3}}} e^{-\frac{r}{a}} e^{i \vec{P} \vec{X}} & \mathrm{H}: \text { hydrogen like } \\
\widetilde{\Psi}=\frac{1}{\sqrt{V_{f}}} \frac{1}{\left(2 \pi \eta^{2}\right)^{3 / 4}} e^{-\frac{r^{2}}{4 \eta^{2}}} e^{i \vec{P} \vec{X}} & \mathrm{G}: \text { gaussian }
\end{array}
$$

These expressions contain the volume normalization factors related to the characteristic volume of quarks $\left(V_{q}\right)$, prehadrons $\left(V_{h}\right)$, and the fireball volume $\left(V_{f}\right)$. 

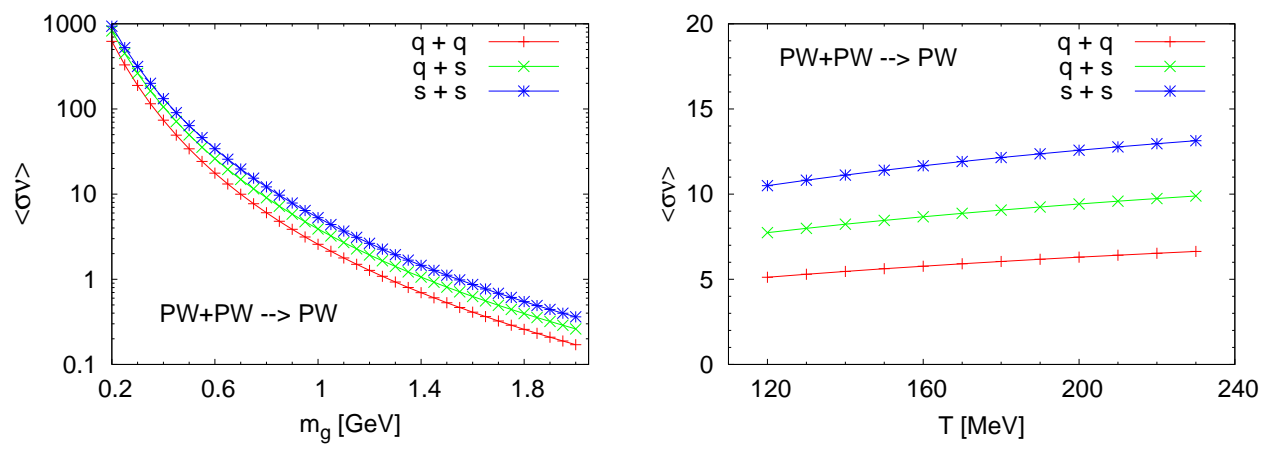

Figure 1. The dependence of the coalescence rate $(\langle\sigma v\rangle)$ on gluon mass $\left(m_{g}\right)$ at fix temperature $(T=180 \mathrm{MeV}$ ) (left) and on temperature ( $\mathrm{T}$ ) at fix gluon mass $\left(m_{g}=800 \mathrm{MeV}\right)$ (right) for the case of quark plain waves coalesce into mesonic plain wave.

The wave functions in eqs.(11)-(12) and (13)-(15) offer us 9 combinations, which can be doubled by the application of Boltzmann and Jüttner momentum distributions. For consistency we will calculate meson and baryon productions in the same (fixed) wave function combination. We note that ALCOR model [1] used the $P W+P W \rightarrow H$ combination, MICOR model [7] applied the $P W+G \rightarrow H$ choice, including a gaussian localization for the picked-up quark.

In the case of process $P W+P W \rightarrow P W$ averaging with the Boltzmann distribution (named as case 'B1'), one can obtain a compact expression for the rate:

$$
\left\langle\sigma^{h} v\right\rangle_{B 1}=\frac{V_{g}^{2} V_{t}}{V_{q}^{2} V_{h}} \frac{M_{h}\left(m_{1}+m_{2}\right)^{2}}{\left(m_{1} m_{2}\right)^{3 / 2}} \frac{\alpha_{s}^{2}}{\pi} \frac{1}{T} \int d k \frac{k^{2} e^{-\frac{m_{1}+m_{2}}{T m_{1} m_{2}} k^{2}}}{\left(k^{2}+m_{g}^{2}\right)^{2}} .
$$

This expression shows that the volume terms can be collected into a prefactor together with the coupling constant $\alpha_{s}$, which is valid for every combinations. This prefactor should be fitted from one data point, and all other particle yields become calculable. On the other hand, this factor will drop out from particle ratios (calculated with the same wave function combination). In the remaining 17 combinations much longer expressions appear for the rates, but all of them can be calculated numerically.

In our investigation we recognized that combinations containing at least one quark plain wave resulted numerically different values, but they differed in a constant factor, only. This is the reason why ALCOR's and MICOR's results on particle yields are generally the same, although different wave function sets have been used.

Now we investigate the sensitivity of the coalescence rate on the mass parameter of the Yukawa potential $\left(m_{g}\right)$ and the temperature $(\mathrm{T})$. In Figure 1 we display the calculated rates (with an arbitrary scale) in the case of $P W+P W \rightarrow P W$, using the expression of eq. (16). One can see (left) that the meson production rates strongly depend on the gluon mass at fix temperature (here we choose $T=180$ $\mathrm{MeV}$ ). Considering the temperature dependence (right), the rates are very much insensitive on this parameter at fix gluon mass $\left(m_{g}=800 \mathrm{MeV}\right)$. This fact verifies the applicability of coalescence models for cross-over phase transitions, where no sharp transition temperature exists. In Figure 1 one can recognize the presence of a flavour dependence, which is connected to the heavier mass of the strangeness. 

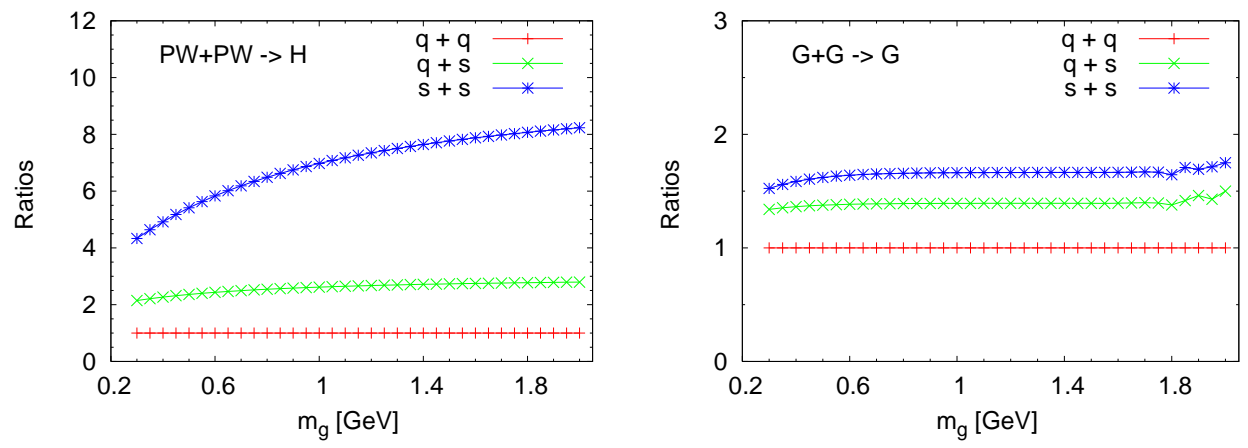

Figure 2. The gluon mass $\left(m_{g}\right)$ dependence of the production ratios of mesons with different flavour combinations, relative to the light meson at fix temperature $(T=180 \mathrm{MeV})$. We display the numerical results for the $P W+P W \rightarrow H$ (left) and $G+G \rightarrow G$ (right).

This flavour dependence is investigated further through the particle ratios, in which case uncertainties connected to unknown volume factors disappear. The temperature dependence of the ratios is negligible, as one could expect from Figure 1. We have found that the strong gluon mass dependence drops out for ratios in all 18 wave function combinations. In Figure 2 we show our numerical results for the case of $P W+P W \rightarrow H$ (left) and the case of $G+G \rightarrow G$ (right), which results illustrate the weak dependence of the ratios on the gluon mass in very different cases.

These results indicate and prove the robustness of the coalescence model within a fixed wave function combination, since the theoretical results depend very weakly on temperature and/or gluon mass. This feature nicely support the applicability of coalescence models to describe cross-over phase transition, which is expected to happen in a wide temperature region.

Now we would like to investigate the sensitivity of the coalescence model on different wave function setups. For this task we want to use real data measured at RHIC energy. Our method is the following: we use one measured meson ratio to fix the open parameters, recalculate other measurable hadron ratios, and investigate the difference between the obtained results in difference wave function setups. One of our candidate for the starting point is the ratio $\Phi / K^{*}=0.60 \pm 0.15$ measured at RHIC in central $\mathrm{Au}+\mathrm{Au}$ collisions at $\sqrt{s}=200 \mathrm{AGeV}$ [14. (We use the middle value and neglect the influence of the error.) In the coalescence model this ratio appears in the following way:

$$
\frac{N_{\phi}}{N_{K^{*}}}=\frac{\left\langle\sigma^{h} v\right\rangle_{s s} \cdot N_{s} N_{s}}{\left\langle\sigma^{h} v\right\rangle_{s q} \cdot N_{s} N_{q}}
$$

The rates in eq. (17) can be calculated in a fixed wave function setup. Thus we can determine the missing $N_{s} / N_{q}$ factor from the measured $N_{\phi} / N_{K^{*}}$ value. Then different strange-non-strange meson and baryon ratios can be calculated from the model. Here we will calculate the ratios $K^{*} / \rho^{0}, \Sigma^{*} / \Delta, \Xi^{*} / \Sigma$ and $\Omega / \Xi^{*}$. These resonances have been measured [15] and the above ratios could be determined from existing experimental data. 
Table 1. Hadron ratios in different wave function setups with Boltzmann (upper part) and Jüttner distributions (lower part). The ratio $N_{s} / N_{q}$ is determined from the fixed ratio $\Phi / K^{*}=0.6$ measured at RHIC [14]. Other particle ratios are calculated from the corresponding wave function setup, using the former strange to light quark ratio.

\begin{tabular}{l|l|llll}
\hline Model & $N_{s} / N_{q}$ & $K^{*} / \rho^{0}$ & $\Sigma^{*} / \Delta$ & $\Xi^{*} / \Sigma$ & $\Omega / \Xi^{*}$ \\
\hline $\mathrm{PW}+\mathrm{PW} \rightarrow \mathrm{PW}$ & 0.448 & 0.671 & 0.790 & 0.700 & 0.644 \\
$\mathrm{PW}+\mathrm{PW} \rightarrow \mathrm{H}$ & 0.235 & 0.598 & 0.848 & 0.785 & 0.746 \\
$\mathrm{PW}+\mathrm{PW} \rightarrow \mathrm{G}$ & 0.474 & 0.686 & 0.791 & 0.690 & 0.628 \\
$\mathrm{G}+\mathrm{G} \rightarrow \mathrm{PW}$ & 0.441 & 0.668 & 0.790 & 0.702 & 0.648 \\
$\mathrm{G}+\mathrm{G} \rightarrow \mathrm{H}$ & 0.227 & 0.593 & 0.849 & 0.791 & 0.753 \\
$\mathrm{G}+\mathrm{G} \rightarrow \mathrm{G}$ & 0.503 & 0.700 & 0.791 & 0.681 & 0.614 \\
\hline $\mathrm{PW}+\mathrm{PW} \rightarrow \mathrm{PW}$ & 0.401 & 0.701 & 0.857 & 0.721 & 0.644 \\
$\mathrm{PW}+\mathrm{PW} \rightarrow \mathrm{H}$ & 0.206 & 0.624 & 0.928 & 0.815 & 0.752 \\
$\mathrm{PW}+\mathrm{PW} \rightarrow \mathrm{G}$ & 0.424 & 0.706 & 0.843 & 0.708 & 0.631 \\
$\mathrm{G}+\mathrm{G} \rightarrow \mathrm{PW}$ & 0.396 & 0.701 & 0.862 & 0.724 & 0.647 \\
$\mathrm{G}+\mathrm{G} \rightarrow \mathrm{H}$ & 0.201 & 0.620 & 0.931 & 0.819 & 0.757 \\
$\mathrm{G}+\mathrm{G} \rightarrow \mathrm{G}$ & 0.435 & 0.712 & 0.845 & 0.706 & 0.627 \\
\hline
\end{tabular}

Table 1. summarizes our numerical results. We can see that different wave function setups result very different $N_{s} / N_{q}$ values, namely 100-150\% difference can be seen between the smallest and the largest values of quark ratios. On the other hand, this uncertainty drops to a 10-15\% difference, both for mesonic and baryonic ratios. These results prove most strikingly why the coalescence models yield very good agreement during data reconstruction, if we start from one measured values. Further analyses are in progress to reveal a deeper connection between the manifestation of conservation laws and the structure of the quantummechanical description of quark coalescence.

This work has been supported in part by the Hungarian OTKA under grants No. NK062044, IN71374, K67942 and the National Natural Science Foundation of China under grant No 10475032.

[1] T.S. Biró, P. Lévai, and J. Zimányi, Phys. Lett. B347, 6 (1995); Phys. Rev. C59, 1574 (1999).

[2] T.S. Biró, T. Csörgö, P. Lévai, and J. Zimányi, Phys. Lett. B472, 243 (2000).

[3] A. Bialas, Phys. Lett. B532, 249 (2002); ibid. B579, 31 (2004).

[4] P. Lévai, U. Heinz, Phys. Rev. C57, 1879 (1998).

[5] T.S. Biró, P. Lévai, J. Zimányi, J. Phys. G25, 311 (1999); ibid. G27, 439 (2001); ibid. G28, 1561 (2002); ibid. G31, 711 (2005).

[6] J. Zimányi, T.S. Biró, T. Csörgő, P. Lévai, Phys. Lett. B472, 243 (2000).

[7] P. Csizmadia and P. Lévai, Phys. Rev. C61, 031903 (2000); Acta Phys. Hung. A22, 371 (2005); ibid. A27, 433 (2006).

[8] P. Csizmadia, P. Lévai, S.E. Vance, T.S. Biró, M. Gyulassy, and J. Zimányi, J. of Phys. G25, 321 (1999).

[9] R.C. Hwa and C.B. Yang, Phys. Rev. C66, 064903 (2002).

[10] V. Greco, C.M. Ko, P. Lévai, Phys. Rev. Lett. 90, 202302 (2003); Phys. Rev. C 68, 034904 (2003).

[11] R.J. Fries, B. Müller, C. Nonaka, S.A. Bass, Phys. Rev. Lett. 90, 202303 (2003); Phys. Rev. C68, 044902 (2003).

[12] D. Molnár, S.A. Voloshin, Phys. Rev. Lett. 91, 092301 (2003); Z.W. Lin, D. Molnar, Phys. Rev. C68, 044901 (2003).

[13] Rearrangement collision, Section 34., in L.I. Schiff: Quantum Mechanics, Second edition, McGraw Hill, New York, 1955.

[14] J. Adams et al., Phys. Rev. C71, 064902 (2005).

[15] B.I. Abelev et al., Phys. Rev. Lett. 97, 132301 (2006). 